\title{
AUTOCUIDADO E RISCO CARDIOMETABÓLICO EM PACIENTES COM HIPERTENSÃO ARTERIAL EM SEGUIMENTO NA ATENÇÃO PRIMÁRIA
}

\author{
Caroline Cristina Menezes Sergio*, Thaís Moreira São-João.
}

\begin{abstract}
Resumo
A Hipertensão Arterial (HA) é uma condição multifatorial caracterizada por níveis pressóricos elevados e sustentados. Constitui relevante fator de riso para o desenvolvimento de doenças cardiovasculares (DCV) e acidente vascular encefálico. Por seu caráter assintomático, tem sido observada baixa adesão ao tratamento e o aparecimento de complicações é exponencial. O autocuidado, por sua vez, tem sido compreendido como um processo de preservação da saúde, por meio de práticas de manutenção da saúde e manejo da doença, sendo, no contexto da HA, adesão a dieta hipossódica, cessação do tabagismo e alcoolismo, prática regular de atividade física (AF), bem como adesão à terapia medicamentosa. Tendo em vista o impacto da HA e a baixa adesão ao tratamento, bem como a importância do autocuidado para o paciente com HA, se faz importante para a Enfermagem conhecer seu processo de autocuidado, dada a relevante ação preventiva-educativa-curativa do enfermeiro. $O$ autocuidado foi avaliado pela versão brasileira da Escala de Autocuidado de Hipertensão (Self-care of Hypertension Inventory - SC-HI) e o risco cardiometabólico pelo escore risco cardiometabólico de Framingham; junto a 23 pacientes em seguimento clínico regular na Atenção Primária à Saúde. Os pacientes com hipertensão arterial atendidos nesta unidade básica de saúde parecem possuir confiança para realizar comportamentos de autocuidado. Sugere-se a condução de estudos futuros com incremento do tamanho amostral, bem como estudos longitudinais que avaliem a manutenção do autocuidado ao longo do tempo.
\end{abstract}

Palavras-chave: enfermagem, autocuidado, hipertensão.

\section{Introdução}

A hipertensão arterial (HA) é um relevante fator de risco para o desenvolvimento de doenças cardiovasculares ${ }^{(1)}$. Seu perfil assintomático contribui para a baixa adesão ao tratamento e o autocuidado, nesse contexto, constitui um processo de preservação da saúde, por meio de práticas de manutenção da saúde e manejo da doença ${ }^{(2)}$.

O objetivo deste estudo foi avaliar o autocuidado e o risco cardiometabólico em pacientes com HA em seguimento na atenção primária.

\section{Resultados e Discussão}

Trata-se de estudo exploratório conduzido em uma Unidade Básica de Saúde do interior do Estado de São Paulo. As informações foram obtidas por meio da versão brasileira da Escala de Autocuidado de Hipertensão (SC$\mathrm{HI})$ e do escore de risco cardiometabólico de Framingham. A amostra $(n=23)$ foi composta por mulheres $(73,9 \%)$, com $61,1(15,4)$ anos, brancas $(47,8 \%)$, vivendo com companheiro $(65,2 \%)$, com renda familiar mensal de $\mathrm{R} \$ 3.042,92$ e 6,4 anos de estudo.

Os sintomas mais prevalentes foram cefaleia $(34,8 \%)$, palpitação $(26,1 \%)$, edema $(26,1 \%)$ e cansaço $(26,1 \%)$. As condições clínicas associadas mais prevalentes foram Diabetes mellitus $(47,8)$ e dislipidemia $(43,5 \%)$.

Os participantes demonstraram níveis satisfatórios de confiança $(74,3(14,9))$ e níveis insatisfatórios para Manutenção $(59,0(15,4))$ e Manejo $(57,4(22,4))$ do Autocuidado, domínios da Escala de Autocuidado; e $38,8 \%$ de chance de desenvolver infarto agudo do miocárdio (IAM) nos próximos 10 anos.

Homens e pretos apresentaram maiores níveis de Confiança. Maior chance de desenvolver um IAM foi observada entre homens e pardos. Não foram encontradas associações significantes entre as demais variáveis.
Tabela 1 - Análise descritiva das medidas da Escala de Autocuidado de Hipertensão e Risco Cardiometabólico entre sujeitos com HA ( $n=23)$. CAMPINAS, 2018.

\begin{tabular}{|c|c|c|}
\hline Variáveis & Média(DP) & Variação \\
\hline $\begin{array}{l}\text { Escala de Autocuidado } \\
\text { de Hipertensão - Domínios }\end{array}$ & & \\
\hline Manutenção & $59,0(15,4)$ & $33,3-84,8$ \\
\hline Manejo & $57,4(22,4)$ & $5,5-94,3$ \\
\hline Confiança & $74,3(14,9)$ & $33,3-99,9$ \\
\hline $\begin{array}{l}\text { Risco cardiometabólico (\%) } \\
\text { Escore de Framinaham }\end{array}$ & $388(164)$ & $1->56$ \\
\hline
\end{tabular}

\section{Conclusões}

Os achados deste estudo permitem concluir que os pacientes atendidos nesta unidade básica de saúde possuem autoconfiança para realizar comportamentos de autocuidado.

\section{Agradecimentos}

PIBIC-CNPq - Bolsa IC - Quota 2017-18

1. Sociedade Brasileira de Cardiologia. VII Diretrizes Brasileiras de Hipertensão Arterial. Revista Brasileira de Hipertensão. 2016; 107(3):1-32

2. Riegel B, Jaarsma T, Strömberg A. A middle-range theory of selfcare of chronic illness. Advances in Nursing Science. 2012; 35(3):194-204 